\title{
Unitary Matrix Models and Phase Transition
}

\author{
Masato HisAKADO \\ Department of Pure and Applied Sciences, \\ University of Tokyo, \\ 3-8-1 Komaba, Megro-ku, Tokyo, 113, Japan
}

May 31, 2021

\begin{abstract}
We study the unitary matrix model with a topological term. We call the topological term the theta term. In the symmetric model there is the phase transition between the strong and weak coupling regime at $\lambda_{c}=2$. If the Wilson term is bigger than the theta term, there is the strong-weak coupling phase transition at the same $\lambda_{c}$. On the other hand, if the theta term is bigger than the Wilson term, there is only the strong coupling regime. So the topological phase transition disappears in this case.
\end{abstract}




\section{Introduction}

Models of the symmetric unitary matrix are solved exactly in the double scaling limit, using orthogonal polynomials on a circle. (1] The partition function is the form $\int d U \exp \left\{-\frac{N}{\lambda} \operatorname{tr}\left(U+U^{\dagger}\right)\right\}$, where $U$ is an $N \times N$ unitary matrix. We call the model symmetric model. 22

This unitary models has been studied in connection with the large- $N$ approximation to QCD in two dimensions.(one-plaquette model) 3] Gross and Witten discovered the third-order phase transition between the weak and strong coupling regime at $\lambda_{c}=2$.

We consider the model which has the symmetric and anti-symmetric part. The symmetric part is the usual Wilson action. The anti-symmetric part becomes the topological term. 四 We can see the topological meaning of the theta term in the continuous limit. it gives rise to a phase transition at $\theta=\pi$, if the Wilson term is bigger than the theta term. [5] We call this phase transition the topological phase transition and the model which has only the theta term the anti-symmetric model.

From the view point of the integrable system this model can be embedded in the two-dimensional Toda hierarchy with the conjugate structure. [6], [7] This Toda equation is split into the modified Volterra (MV) equation and the discrete nonlinear Schrödinger (DNLS) equation. MV and DNLS equations correspond to the symmetric and anti-symmetric model respectively. Coupling the Toda equation and the string equation we can derive the third Painlevé (P III) equation. We use P III to study the phase structure.

This letter is organized as follows. In the section 2 we introduce the unitary matrix model with a topological term. We consider the two cases:

(i) the Wilson term is bigger than the theta term,

(ii) the theta term is bigger than the theta term.

In the section 3 we consider the phase transition in the case (i) using the $\mathrm{P}$ III. In the section 4 we study the phase structure in the case (ii). The last 
section is devoted to the concluding remarks.

\section{Wilson term and theta term}

We consider the partition function of the unitary matrix model with a topological term. We consider the unitary matrix model

$$
Z_{N}=\int d U \exp \left(-\frac{N}{\lambda} V(U)\right)
$$

where $V(U)$ is a potential

$$
V(U)=t_{1} U+t_{-1} U^{\dagger}
$$

$U$ is the gauge group $U(N)$.

We divide the potential into the symmetric and the anti-symmetric part,

$$
V(U)=t_{+} s_{w}+t_{-} s_{\theta}
$$

where

$$
t_{+}=\frac{t_{1}+t_{-1}}{2}, \quad t_{-}=\frac{t_{1}-t_{-1}}{2} .
$$

$s_{w}$ is the symmetric part, the usual Wilson action

$$
s_{w}=\frac{1}{2}\left(\operatorname{tr} U+\operatorname{tr} U^{\dagger}\right)
$$

Here we choose

$$
s_{\theta}=\frac{1}{2}\left(\operatorname{tr} U-\operatorname{tr} U^{\dagger}\right)
$$

for the theta term, the anti-symmetric part.

Hereafter we call next reduced models the symmetric model and the antisymmetric model:

$$
\begin{aligned}
& t_{1}=t_{-1}=t_{+}, \quad t_{-}=0, \quad(\text { symmetric model }) \\
& t_{1}=-t_{-1}=t_{-}, \quad t_{+}=0 . \quad(\text { anti }- \text { symmetric model })
\end{aligned}
$$


Here we consider the two cases

$$
\text { (i) }\left|t_{+}\right|>\left|t_{-}\right|, \quad \text { (ii) }\left|t_{-}\right|>\left|t_{+}\right| \text {. }
$$

We parameterize $t_{1}$ and $t_{-1}$ by $\epsilon$ :

$$
\begin{aligned}
& \text { (i) } t_{1}=-e^{\epsilon}, \quad t_{-1}=-e^{-\epsilon}, \\
& \text { (ii) } t_{1}=-e^{\epsilon}, \quad t_{-1}=e^{-\epsilon} .
\end{aligned}
$$

The measure $d U$ may be written as

$$
d U=\prod_{m}^{N} \frac{d \alpha_{m}}{2 \pi} \Delta(\alpha) \bar{\Delta}(\alpha) .
$$

Here the eigenvalues of $U$ are $\left\{\exp \left(i \alpha_{1}\right), \exp \left(i \alpha_{2}\right), \cdots, \exp \left(i \alpha_{N}\right)\right\}$ and $\Delta \bar{\Delta}$ is the Jacobian for the change of variables,

$$
\begin{aligned}
& \Delta(\alpha)=\operatorname{det}_{j k} e^{i \alpha_{j}(N-k)}, \\
& \bar{\Delta}(\alpha)=\operatorname{det}_{j k} e^{-i \alpha_{j}(N-k)} .
\end{aligned}
$$

Then we obtain the partition function in the case (i):

$$
Z_{N}=\text { const.det }_{j k} e^{\epsilon(-j+k)} I_{-j+k}(N / \lambda)={\text { const. } \operatorname{det}_{j k} I_{-j+k}(N / \lambda)}
$$

Here $I_{-j+k}$ is the modified Bessel function of order $-j+k$. In the same way we can calculate the partition function in the case (ii):

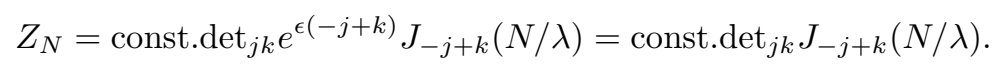

Here $J_{-j+k}$ is the Bessel function of order $-j+k$. Notice that (2.10) and (2.11) do not depend on $\epsilon$.

It is well known that the partition function $Z_{N}$ of the unitary matrix model can be presented as a product of norms of the biorthogonal polynomial system. Namely, let us introduce a scalar product of the form

$$
<A, B>=\oint \frac{d \mu(z)}{2 \pi i z} \exp \{-V(z)\} A(z) B\left(z^{-1}\right)
$$


where

$$
V(z)=t_{1} z+t_{-1} z^{-1}
$$

Let us define the system of the polynomials biorthogaonal with respect to this scalar product

$$
<\Phi_{n}, \Phi_{k}^{*}>=h_{n} \delta_{n k}
$$

Then, the partition function $Z_{N}$ is equal to the product of $h_{n}$ 's:

$$
Z_{N}=\prod_{k=0}^{N-1} h_{k}, \quad Z_{0}=1 .
$$

The polynomials are normalized as follows (Note that the asterisk ' $*$ ' does not mean the complex conjugation):

$$
\Phi_{n}=z^{n}+\cdots+S_{n-1}, \quad \Phi_{n}^{*}=z^{n}+\cdots+S_{n-1}^{*}, \quad S_{-1}=S_{-1}^{*} \equiv 1 .
$$

Now it is easy to show that these polynomials satisfy the following recurrent relations,

$$
\begin{aligned}
\Phi_{n+1}(z) & =z \Phi_{n}(z)+S_{n} z^{n} \Phi_{n}^{*}\left(z^{-1}\right), \\
\Phi_{n+1}^{*}\left(z^{-1}\right) & =z^{-1} \Phi_{n}^{*}\left(z^{-1}\right)+S_{n}^{*} z_{-n} \Phi_{n}(z),
\end{aligned}
$$

and

$$
\frac{h_{n+1}}{h_{n}}=1-S_{n} S_{n}^{*}
$$

From (2.14) we can obtain the string equations:

$$
\begin{aligned}
& (n+1) S_{n}=\left(t_{-1} S_{n+1}+t_{1} S_{n-1}\right)\left(1-S_{n} S_{n}^{*}\right), \\
& (n+1) S_{n}^{*}=\left(t_{1} S_{n+1}^{*}+t_{-1} S_{n-1}^{*}\right)\left(1-S_{n} S_{n}^{*}\right) .
\end{aligned}
$$

In the unitary matrix model there is a conjugate relation:

$$
t_{1} S_{n} S_{n-1}^{*}=t_{-1} S_{n}^{*} S_{n-1}
$$


Here we define $a_{n}$ :

$$
a_{n} \equiv 1--S_{n} S_{n}^{*}=\frac{h_{n+1}}{h_{n}} .
$$

From (2.20) $a_{n}$ are functions of the radial coordinate

$$
x=t_{1} t_{-1}
$$

only.

$a_{n}$ satisfies the next Painlevé $\mathrm{V}$ with $\delta_{V}=0:[7$

$$
\begin{aligned}
\frac{\partial^{2} a_{n}}{\partial x^{2}}= & \frac{1}{2}\left(\frac{1}{a_{n}-1}+\frac{1}{a_{n}}\right)\left(\frac{\partial a_{n}}{\partial x}\right)^{2}-\frac{1}{x} \frac{\partial a_{n}}{\partial x} \\
& -\frac{2}{x} a_{n}\left(a_{n}-1\right)+\frac{(n+1)^{2}}{2 x^{2}} \frac{a_{n}-1}{a_{n}} .
\end{aligned}
$$

\section{Phase structure in the case (i)}

The partition function dose not depend on $\epsilon$ from (2.10). This can be seen from the radial coordinate $(2.22)$. From these results in the large- $N$ limit the phase structure in the case (i) is the same as the symmetric model. [3] To study the strong-weak coupling phase transition we use (2.23) in $x \rightarrow \infty$. We rewrite (2.23) a second order ODE for $S_{n}$

$$
\frac{\partial^{2} S_{n}}{\partial t_{+}^{2}}=-\frac{S_{n}}{1-S_{n}^{2}}\left(\frac{\partial S_{n}}{\partial t_{+}}\right)^{2}-\frac{1}{t_{+}} \frac{\partial S_{n}}{\partial t_{+}}+\frac{(n+1)^{2}}{t_{+}^{2}} \frac{S_{n}}{1-S_{n}^{2}}-4 S_{n}\left(1-S_{n}^{2}\right),
$$

where $t_{+}=N / \lambda$. This equation can be obtained directly from coupling the string equation and the modified Volterra equation.[7]

In particular when we consider the strong coupling regime we only need to solve

$$
\frac{\partial S_{n}}{\partial t_{+}^{2}}+\frac{1}{t_{+}} \frac{\partial S_{n}}{\partial t_{+}}-\left[\frac{(n+1)^{2}}{t_{+}^{2}}-4\right] S_{n}=0 .
$$

This is the Bessel equation. Then setting $n=N$, we can obtain in the large- $N$ limit

$$
S_{N}=J_{N}\left(2 t^{+}\right)+O\left(\frac{1}{\lambda^{(3 N+2)}}\right) \stackrel{N \rightarrow \infty}{\longrightarrow} J_{N}(2 N / \lambda)
$$


where $J_{N}$ is the standard Bessel function. As a consequence, we find

$$
\left.S_{N} \sim \exp N\left[\sqrt{1-\frac{4}{\lambda^{2}}}-\log \frac{\lambda\left(1+\sqrt{1-4 / \lambda^{2}}\right.}{2}\right)\right] .
$$

(3.1) is especially appropriate for a discussion of the weak coupling regime. One may consider an $1 / N$ expansion for $S_{N}$ : [8]

$$
S_{N} \rightarrow \sqrt{1-\frac{\lambda}{2}}-\frac{1}{N^{2}} \frac{\lambda^{3}}{128}\left(1-\frac{\lambda}{2}\right)^{5 / 2}+O\left(\frac{1}{N^{4}}\right) .
$$

We notice the typical phase transition behavior of $S_{N}$. The critical point $\lambda_{c}=2$ is independent of the parameter $\epsilon$.

\section{Phase structure in the case (ii)}

In the large $N$-limit to study the phase structure we use the string equations (2.19a and (2.19b). Setting $n=N$, there is a critical point at $\lambda_{c}=2 \sinh \epsilon$ for the roots of the differential equation are degenerate. It seems that in the limit $\epsilon \rightarrow 0$ (the Wilson term vanishes) the weak coupling regime disappears. But as seeing in the previous section this is not correct. From the radial coordinate or (2.11) the critical point does not depend on $\epsilon$. The phase structure in the case (ii) is the same as the anti-symmetric model. To see the phase structure we study (2.23) in $x \rightarrow-\infty$. Then the difference between the case (i) and (ii) is the sign of the third term of RHS of (2.23). We rewrite (2.23) a second order ODE for $S_{n}$ :

$$
\frac{\partial^{2} S_{n}}{\partial t_{-}^{2}}=-\frac{S_{n}}{1-S_{n}^{2}}\left(\frac{\partial S_{n}}{\partial t_{-}}\right)^{2}-\frac{1}{t_{-}} \frac{\partial S_{n}}{\partial t_{-}}+\frac{(n+1)^{2}}{t_{-}^{2}} \frac{S_{n}}{1-S_{n}^{2}} \quad \begin{aligned}
& 4 S_{n}\left(1-S_{n}^{2}\right) \\
& (n=\text { odd })(4.1 \mathrm{a})
\end{aligned}
$$

and

$$
\frac{\partial^{2} S_{n}}{\partial t_{-}^{2}}=-\frac{S_{n}}{1+S_{n}^{2}}\left(\frac{\partial S_{n}}{\partial t_{-}}\right)^{2}-\frac{1}{t_{-}} \frac{\partial S_{n}}{\partial t_{-}}+\frac{(n+1)^{2}}{t_{-}^{2}} \frac{S_{n}}{1+S_{n}^{2}} \quad \begin{aligned}
& 4 S_{n}\left(1+S_{n}^{2}\right), \\
& (n=\text { even })(4.1 \mathrm{~b})
\end{aligned}
$$


using

$$
\begin{aligned}
& S_{n}=S_{n}^{*}, \quad a_{n}=1-S_{n}^{2}, \quad(n=\text { odd }), \\
& S_{n}=-S_{n}^{*}, \quad a_{n}=1+S_{n}^{2}, \quad(n=\text { even }) .
\end{aligned}
$$

Notice $t_{-}=2 N / \lambda$. This equation can be obtained directly from the coupling the string equations and the discrete nonlinear Schrödinger equation. 7 When we consider the strong coupling regime we only need to solve

$$
\frac{\partial S_{n}}{\partial t_{-}^{2}}+\frac{1}{t_{-}} \frac{\partial S_{n}}{\partial t_{-}}-\left[\frac{(n+1)^{2}}{t_{-}^{2}}+4\right] S_{n}=0 .
$$

This is the modified Bessel equation. Then setting $n=N$, we can obtain in the large- $N$ limit

$$
S_{N}=K_{N}\left(2 t_{-}\right)+O\left(\frac{1}{\lambda^{(3 N+2)}}\right) \stackrel{N \rightarrow \infty}{\longrightarrow} K_{N}(2 N / \lambda),
$$

where $K_{N}$ is the second kind modified Bessel function. As a consequence, we obtain

$$
S_{N} \sim \exp N \sqrt{1+\frac{4}{\lambda^{2}}} .
$$

On the other hand we can not do an $1 / N$ expansion in (4.1a) and 4.1b), since there is not the weak coupling regime. Then there is not the strong-weak coupling phase transition in the case (ii).

In the previous letter, [5] adding the term $l \log U$ we have shown that there is the phase transition at $\theta=\pi$ in the case (i). We call the phase transition the topological phase transition. This phase transition can be seen in the weak coupling regime. [4] So in the case (ii) there is not the topological phase transition, too.

\section{Concluding remarks}

We study the unitary matrix model with a topological term. This model contains the Wilson term and the theta term. The Wilson term is the symmetric and the theta is the anti-symmetric part. We call the model which has only the 
Wilson term the symmetric model and the model which has only the theta term the anti-symmetric model. It is well known that in the symmetric model there is the strong-weak coupling phase transition at $\lambda_{c}=2$. If the Wilson term is bigger than the theta term, there is the strong-weak coupling phase transition at the same $\lambda_{c}$. Adding the term $l \log U$, there is the topological phase transition at $\theta=\pi$. On the other hand if the theta term is bigger than the Wilson term, there is no strong-weak coupling phase transition. Since there is only the strong coupling regime, the topological phase transition also disappears.

\section{References}

[1] V.Periwal and D.Shevitz, Phys.Rev.Lett.64(1990)1326.

[2] M.Bowick, A.Morozov and D.Shevitz, Nucl.Phys.B354(1991)496.

[3] D.Gross and E.Witten, Phys.Rev.D.21(1980)446.

[4] T.G.Koács, E.T.Tomboulis, Z.Schram, Nucl.Phys.B454(1995)45.

[5] M.Hisakado, Phys.Lett.B 395(1997)208.

[6] S.Kharchev, A.Marshakov, A.Mironov, A.Orlov and A.Zabrodin, Nucl.Phys.B366(1991)569.

[7] M.Hisakado, Mod.Phys.Lett.A 38(1996)3001.

[8] A.Guha and S.-C.Lee, Nucl.Phys.B240(1984) 141. 